\title{
Présentation de la campagne de mesures intensives du PNEC : Truc Vert 2001
}

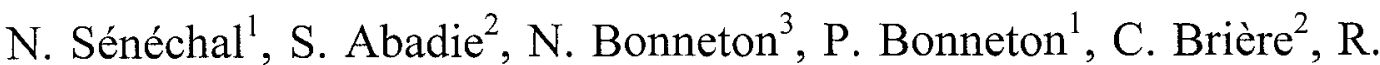
Butel $^{1}$, R. Capobianco ${ }^{4}$, B. Castelle ${ }^{1}$, G. Chapalain ${ }^{5}$, F. Desmazes ${ }^{1}$, C. Dulou $^{1}$, H. Dupuis ${ }^{1}$, H. Howa ${ }^{6}$, P. Larroude ${ }^{7}$, P. Maron ${ }^{2}$, D. Malengros $^{5}$, D. Michel ${ }^{1}$, M. Mory ${ }^{8}$, G. Oggian ${ }^{1}$, R. Pedreros ${ }^{1}$, V. Rey ${ }^{4}$, D. Rihouey ${ }^{2}$

${ }^{1} D G O /$ UMR CNRS EPOC, Univ. Bordeaux I. n.senechal@geocean.u-bordeaux.Fr

${ }^{2}$ LASAGEC, Univ. de Pau et des Pays de l'Adour.

${ }^{3}$ MASTER, ENSCPB, Univ. Bordeaux I.

${ }^{4}$ Univ. Toulon et du Var.

${ }^{5}$ LSG, Univ. de Lille I.

${ }^{6}$ Univ. D'Angers.

${ }^{7}$ LEGI, Univ. de Grenoble I.

${ }^{8}$ ENSGTI, Univ. de Pau et des Pays de l'Adour.

Résumé

Cet article rassemble les résultats d'une campagne de mesures qui a été réalisée sur la plage aquitaine du Truc Vert du 14 au 19 octobre 2001. Différentes techniques et méthodologies expérimentales ont été mises en œuvre pour caractériser l'hydrodynamique, le transport sédimentaire, ainsi que l'évolution morphologique de cette plage sableuse.

Abstract

The aim of this paper is a field experiment that has been undertaken on the aquitain Truc Vert beach from the $14^{\text {th }}$ to the $19^{\text {th }}$ October 2001. Different experimental techniques and methodologies were implemented for the characterization of hydrodynamics, sediment transport and morphological evolution of this sandy beach.

\section{Introduction}

Dans le cadre de l'action de recherche «Hydrodynamique Sédimentaire » du Programme National d'Environnement Côtier (PNEC), une campagne de mesures a été réalisée sur la plage aquitaine du Truc Vert du 14 au 19 octobre 2001. L'objectif principal de cette action de recherche est d'avancer dans la compréhension et la modélisation des processus qui contrôlent l'évolution morphologique des corps sableux littoraux. La campagne Truc Vert 2001 avait pour objectif plus spécifique d'étudier l'hydrodynamique en milieu peu profond associée à des houles énergétiques et de comprendre son impact sur l'évolution 
morphodynamique des systèmes de barres sableuses que l'on observe sur une côte linéaire à régime meso-tidal et forcée par des houles océaniques. Cette expérience a réuni 22 chercheurs venant de 8 laboratoires français, dont les compétences couvrent aussi bien le domaine des mesures hydrodynamiques, des mesures sédimentaires, que la caractérisation de la morphologie des corps sableux. Cette expérience a été définie en liaison étroite avec les chercheurs du PNEC travaillant sur la modélisation des processus hydro-sédimentaires littoraux, mais aussi en interaction avec le groupe de travail « Hydrodynamique de la zone affectée par le déferlement bathymétrique » du programme national PATOM (Programme ATmosphère Océan à Multi-échelles).

\section{Site expérimental et forcage hydrodynamique}

La plage du Truc Vert est située à environ $10 \mathrm{~km}$ au Nord de la pointe du Cap Ferret et à $90 \mathrm{~km}$ au sud de l'embouchure de l'estuaire de la gironde (cf. figure 1). C'est une plage méso à macrotidale composée de sable moyen et soumise à une houle océanique, principalement de secteur $\mathrm{O} / \mathrm{NO}$. Pendant la période hivernale, les hauteurs significatives sont de l'ordre de $1,55 \mathrm{~m}$ pour une période significative de $12 \mathrm{~s}$ alors qu'en conditions estivales, les hauteurs significatives sont de l'ordre de $0,9 \mathrm{~m}$ pour un période de $7 \mathrm{~s}$. Pour ce qui est des conditions extrêmes, la période de retour de vagues de hauteurs significatives de $8 \mathrm{~m}$ est de l'ordre de 10 ans (Butel et coll. [2]).

La plage du Truc Vert est généralement constituée de deux systèmes de barres sableuses :

- une barre pré-littorale festonnée située à 400 ou $450 \mathrm{~m}$ de la laisse de basse mer. Sa longueur d'onde moyenne est de $1000 \mathrm{~m}$. La partie parallèle au rivage se situe à environ $4,5 \mathrm{~m}$ de profondeur tandis que les cornes se trouvent sous $2 \mathrm{~m}$ d'eau (Desmazes et coll. [3]).

- un système de barre et baïne ("ridge et runnel") en zone intertidale dont la morphologie est extrêmement variable (Michel et Howa [5]). Les barres internes sont, en conditions estivales, allongées vers le sud et interrompues par les baïnes (dépressions peu profondes) tous les $420 \mathrm{~m}+/-160 \mathrm{~m}$.

Sur la photographie aérienne du Truc Vert prise à marée basse pendant la campagne de mesure (figure 2) on peut distinguer les systèmes de barres et baïnes en zone intertidale et de façon indirecte les barres en croissant, dont les cornes sont associées aux zones de déferlement régulièrement espacées.

Les conditions expérimentales observées pendant la campagne correspondent à une période de beau temps (vent faible, hautes pressions atmosphériques supérieures ou égales à $1000 \mathrm{Hpa}$ et absence de précipitations). Les états de mer sont cependant énergétiques (cf. figure 3) avec de longues périodes et des hauteurs significatives atteignant $3 \mathrm{~m}$ (le 18/10). Ces vagues correspondent à des houles formées au large. 


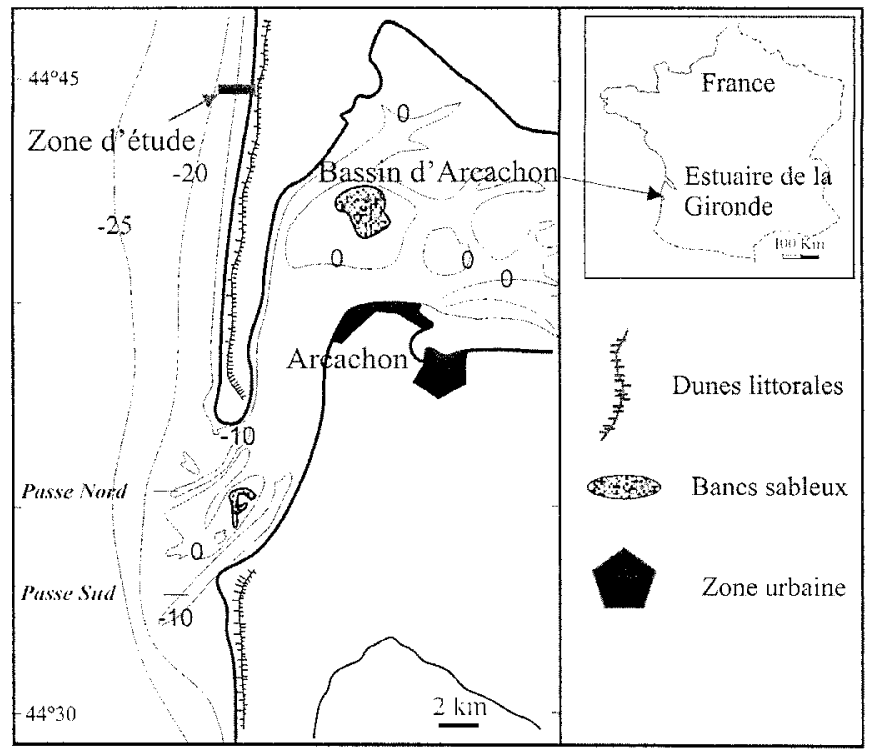

Figure 1. Situation de la zone d'étude Figure 1. Location of the study area

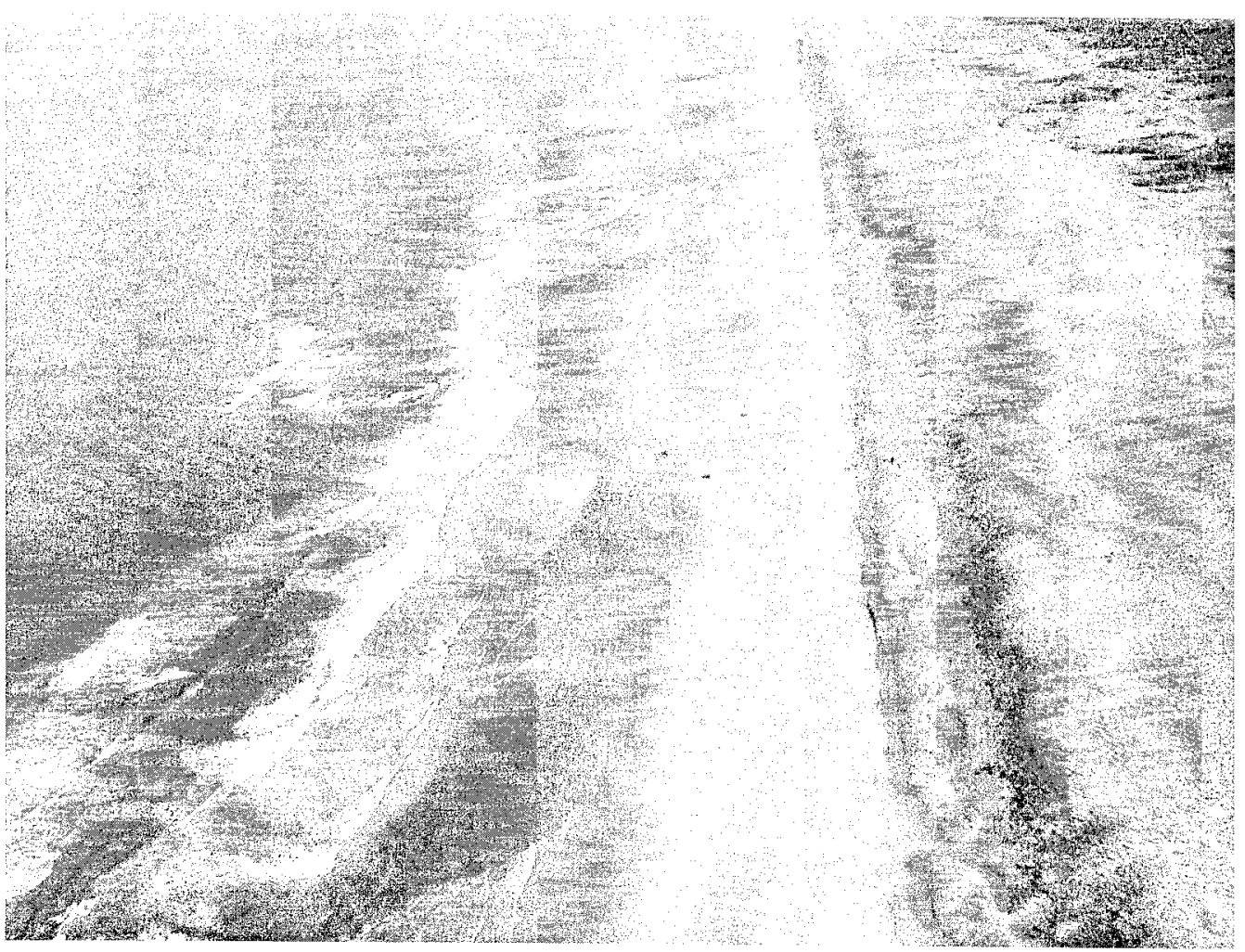

Figure 2. Photographie aérienne de la plage du Truc Vert le 15/10/01 (8h TU) Figure 2. Aerial photograph of the Truc Vert beach 15/10/01 (8h UT) 

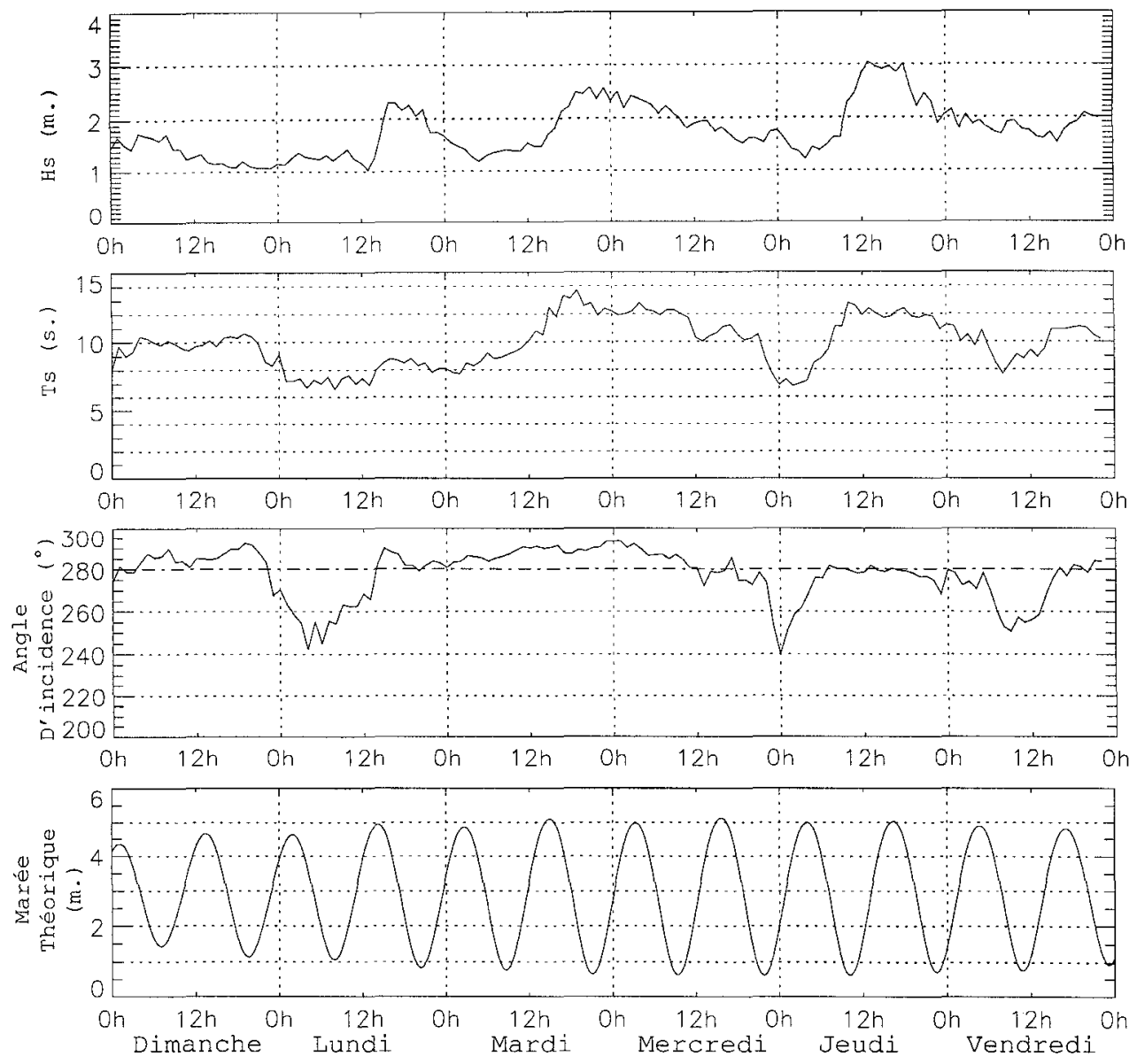

Figure 3. Hauteur significative (Hs), période significative (Ts) et angle de direction moyenne de la houle et variation tidale de la hauteur du plan d'eau du $14 / 10 / 01$ au $19 / 10 / 01$

Figure 3. Significant wave height $(H s)$, period and wave direction and the tidal height variation from 14/10/01 to 19/10/01

\section{3. $\underline{\text { Objectifs }}$}

L'objectif général de cette campagne de mesures est d'étudier les processus hydrodynamiques associés à la transformation de houles énergétiques sur une plage sableuse et tout particulièrement en zone de surf et de comprendre leur impact sur l'évolution morphologique de cette plage. Cette expérience à courte échelle de temps est complémentaire des études long terme réalisées au DGO et qui font la connexion entre la climatologie de la houle (Butel et coll. [2]) et l'évolution morphologique des plages sableuses girondines (Lafon et coll. [4]).

La campagne Truc Vert 2001 se situe aussi dans la continuité de celles déjà réalisées dans le cadre du PNEC en 1998 et 1999 (cf. Michel et coll. [6], 
Sénéchal et coll. [7] et Bonneton et Dupuis [1]), ces deux premières expériences ayant soulevé plusieurs questions :

- Quel est l'impact de la libération des harmoniques de la houle au passage d'une barre, sur la dissipation de ces vagues et par conséquence sur l'évolution morphodynamique du haut de plage (Sénéchal et coll. [8])?

- Quelle est l'influence du phénomène d'appariement des fronts d'onde sur la dissipation des vagues et donc sur le taux de production de turbulence en zone de surf interne et en zone de swash (Sénéchal et coll. [7] et Bonneton et Dupuis [1]) ? - Comment caractériser le critère de déferlement suivant que l'on se trouve sur une barre ou sur une pente de plage régulière.

D'autre part, l'intégration de nouvelles équipes et le renforcement depuis deux ans du potentiel expérimental de l'ensemble des participants, a permis d'aborder des problèmes qui ne pouvaient l'être lors des deux précédentes expériences. En particulier, si en 1998 et 1999 nous avons principalement étudié l'évolution cross-shore de l'hydrodynamique, en revanche en 2001 nous avons pu installer un réseau d'instruments suffisamment dense pour caractériser aussi la variabilité longshore de l'hydrodynamique (houle, courants moyens et ondes infragravitaires), ainsi que le transport sédimentaire en suspension associé au déferlement Cette expérience était aussi la première tentative sur ce site pour quantifier le transport sédimentaire à une échelle temporelle plus proche de celle utilisée pour l'hydrodynamique $(<12 \mathrm{~h})$, que ce soit par vidéo, ou par échantillonnage de la concentration de sédiment en suspension.

\section{Dispositif expérimental et méthodes}

Durant cette campagne de mesures, des méthodes expérimentales permettant de caractériser et quantifier l'évolution morphologique de la plage à différentes échelles spatiales ont été associées à des mesures hydrodynamiques et sédimentaires à haute fréquence d'acquisition.

\subsection{Caractérisation de la morphologie des corps sableux}

\section{Mesures topographiques à l'aide d'un système GPS cinématique temps réel}

Le laboratoire LASAGEC a réalisé à chaque marée basse un relevé topographique de la zone intertidale, sur une distance de $1,5 \mathrm{~km}$, à l'aide d'un système DGPS (cf. figure 4). Le système utilisé est un THALES SCORPIO 6502 MK. Ce DGPS cinématique bi-fréquence est basé sur une méthode cinématique qui rend techniquement possible sur de larges zones $(40 \mathrm{~km}$ autour de la station de référence), un positionnement centimétrique en temps réel. La station de référence a été positionnée exactement par rapport à une borne IGN située dans les dunes de la plage du Truc Vert. 


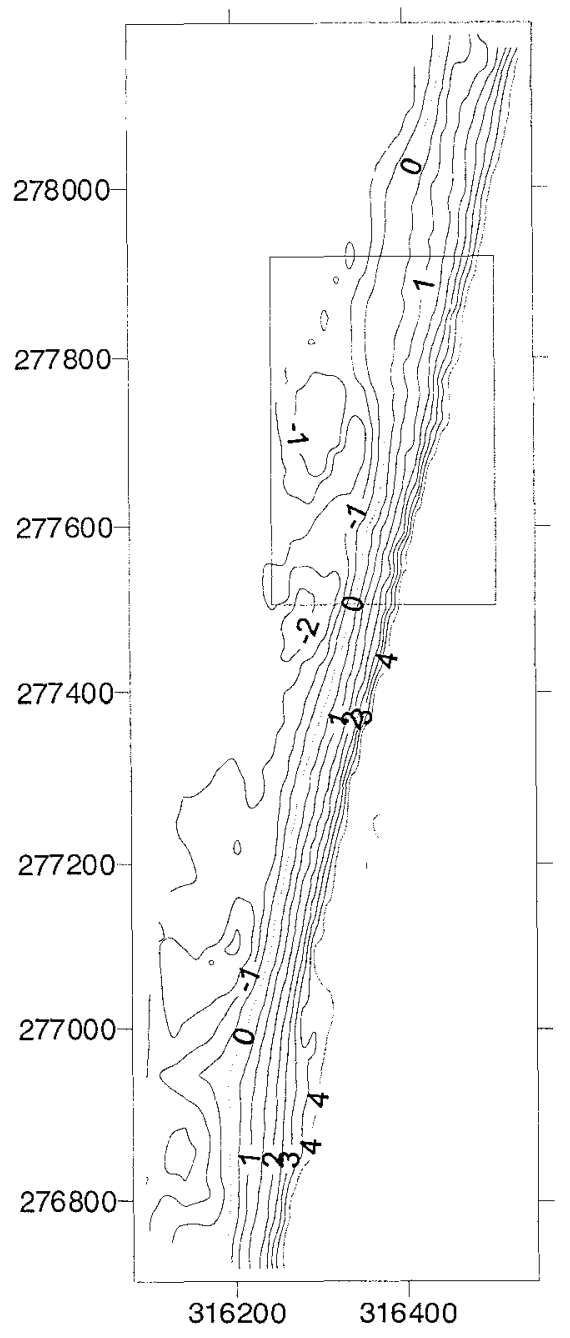

ligne de capteurs de pression 1 ligne de capteurs de pression 2 ligne de capteurs de pression 3

$\oplus \mathrm{S} 4$

$\boxplus$ doppler

$\triangle \mathrm{ADV}$

station SAMBA

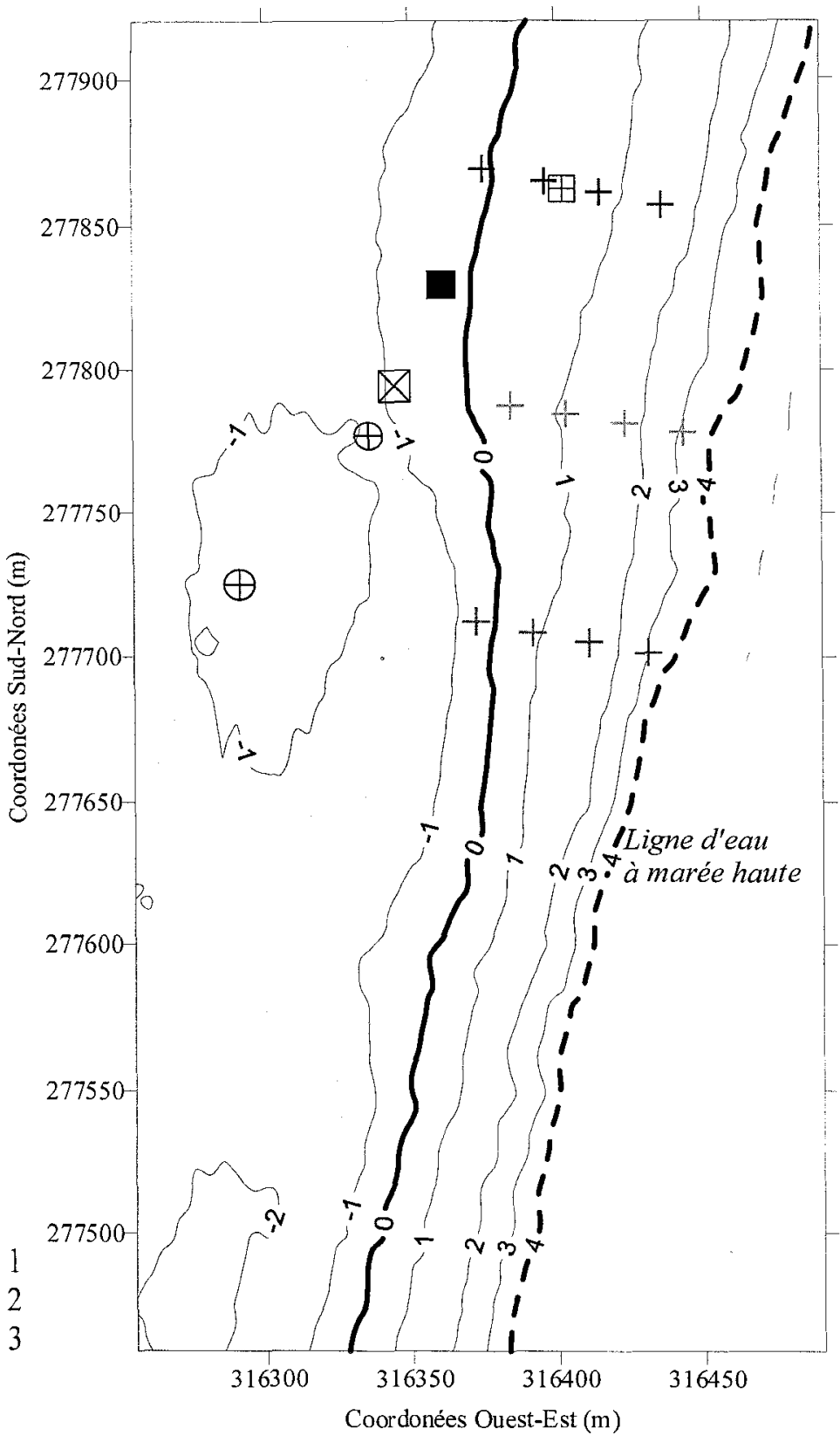

Figure 4. Topographie de la zone interdidale et implantation des instruments Figure 4. Topography of the intertidal zone and instrument locations

Des résultats préliminaires sont présentés sur la figure 5. La topographie de la zone instrumentée permet de distinguer un système de barre-baïne ainsi qu'une berme bien développée le 16/10. Les changements morphologiques enregistrés par les relevés du 18/10 montrent qu'au cours de cette campagne de terrain, la berme s'est érodée jusqu'à 1,30 m localement. La partie moyenne de l'estran présente une 
accrétion globale de l'ordre de $0,20 \mathrm{~m}$ et la barre intertidale migre de $25 \mathrm{~m}$ vers le haut de plage.
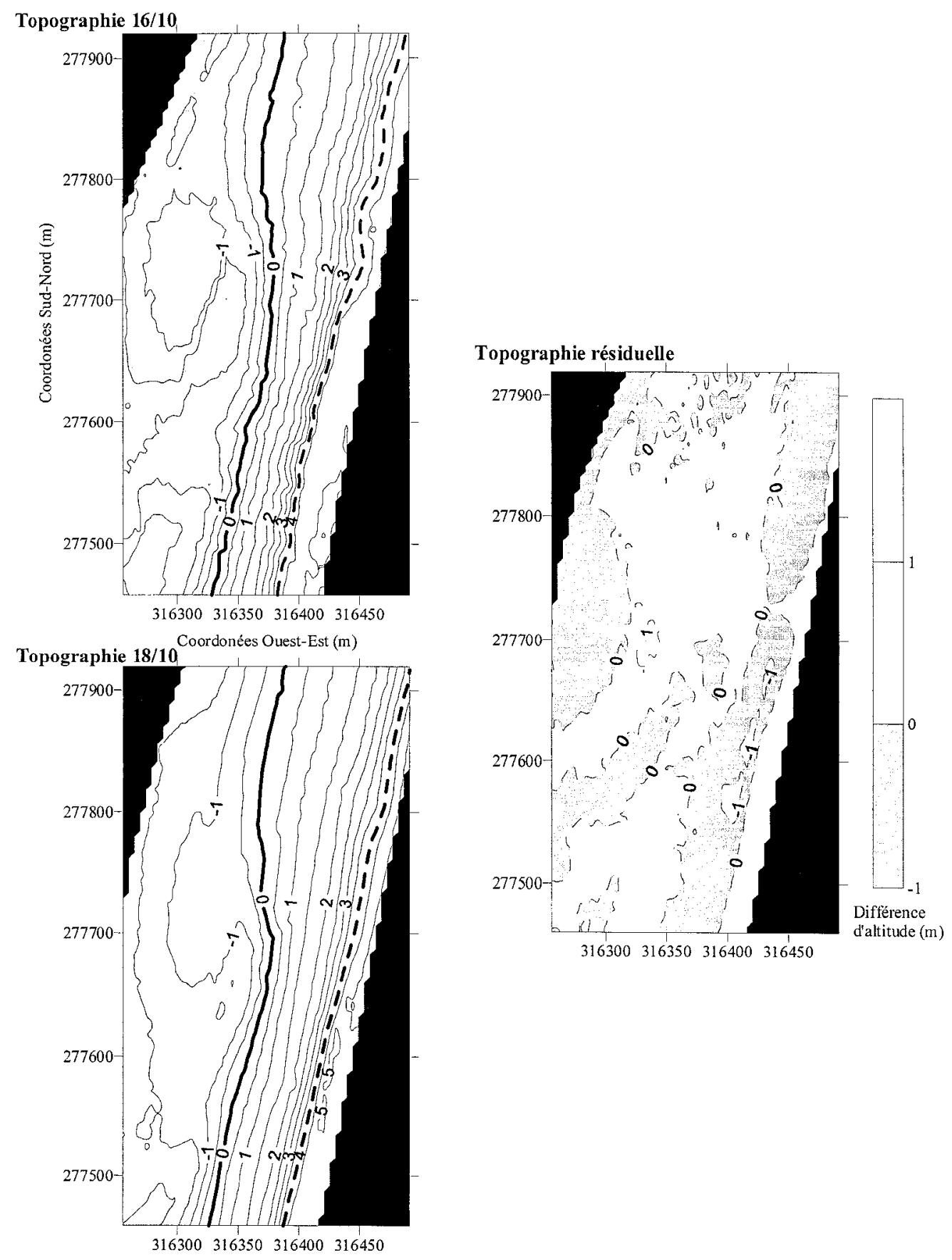

Figure 5. Changements topographiques de la zone intertidale entre le 16/10 et le 18/10. Figure 5. Topographic changes of the intertidal zone between $16 / 10$ and $18 / 10$ 


\section{Système vidéo}

L'un des moyens efficaces pour observer les changements morphologiques côtiers à haute fréquence et à grande échelle temporelle est de recourir à l'implantation permanente de systèmes vidéo. Depuis son invention, la technologie a évolué afin d'obtenir une calibration fine des caméras et permet d'obtenir par stéréoscopie la transformation de vues obliques en images planes non déformées. Ces images consistent typiquement à utiliser l'enregistrement continu de la position du déferlement comme proxy de la morphologie des barres sous-marines. Elles permettent de cartographier la topographie de la zone intertidale et d'estimer les caractéristiques des houles incidentes (hauteur, période, longueur d'onde et angle d'incidence à la côte).

Les premiers tests de cette méthode ont été effectués pendant cette campagne de mesures. Une caméra numérique a été implantée au sommet d'un échafaudage lui-même installé au sommet de la dune. La hauteur de l'objectif de la caméra par rapport au niveau d'eau à basse mer était de $28,50 \mathrm{~m}$. Un système de cibles au sol géoréférencé et visible sur toutes les prises de vue permet une correction géométrique des images obtenues. Cinq prises de vues de 10 minutes ont été effectuées à marée basse. Elles permettront la cartographie des zones moyennes de déferlement sur plus de $800 \mathrm{~m}$ de large. De plus, des plans de 10 min. pris à chaque heure seront utilisés pour mieux comprendre le déplacement avec la marée de la zone de déferlement sur les structures sédimentaires.

\section{Photographies et vidéos aériennes}

Un survol du site a été réalisé le lundi 15 octobre pendant la marée basse du matin. Les photographies qui ont été prises (http://legi.hmg.inpg.fr/houle/PhotoAer.pdf) permettront en particulier de caractériser les systèmes de barres et baïnes tout le long de la pointe sableuse du Cap Ferret. Dès à présent ces photos mettent en évidence la présence de beach cusps au niveau de la laisse de haute mer.

\section{Images SPOT}

Une programmation d'images SPOT multi-spectrales haute résolution a permis d'obtenir trois prises de vues sans couverture nuageuse, centrées sur la plage du Truc Vert, les 07/10/01, 16/10/01 et 30/10/01. Les images du 07/10 et du 30/10 nous permettront de caractériser l'état des systèmes de barres avant et après notre campagne de mesures. De plus, les conditions très favorables (beau temps, très faible houle) du 30/10 devrait nous permettre d'obtenir une bathymétrie des zones de petits fonds, à partir de la méthode mise au point par Lafon et coll. [4]. L'image du 16/10 a été obtenue pendant l'expérience et pour des houles très régulières de fortes amplitudes $\left(\mathrm{Hs}_{\mathrm{s}}=2 \mathrm{~m}\right)$. Cette image, couplée aux données hydrodynamiques, nous permettra de mieux comprendre la propagation de la houle du plateau continental jusqu'à la ligne d'eau et en particulier la réfraction de la houle et la localisation du déferlement sur les 


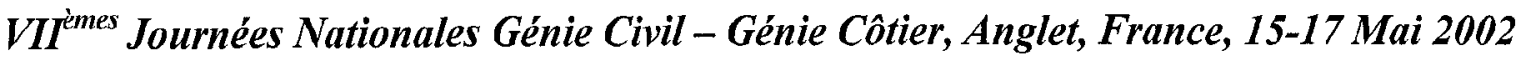

systèmes de barres. Ce travail a pour objectif une amélioration des modèles de propagation de la houle à moyenne échelle, tel que le code SWAN.

\subsection{Mesures hydrodynamiques et sédimentaires}

\section{Mesures de la houle et des courants}

Le DGO a installé en août 2001, en collaboration avec le CETMEF, une bouée TRIAXYS constituée d'un flotteur de surface munis d'accéléromètres et permettant de calculer le spectre directionnel des vagues par $-54 \mathrm{~m}$ de fond, soit en conditions de forçage au large. Des données simplifiées de cette bouée son disponibles en temps réel sur le site http://www.equipement.gouv.fr/cetmef/candhis/. Par ailleurs, ces mesures sont couplées à celles d'une bouée houle DATAWELL distante de $120 \mathrm{~m}$ de la TRIAXYS pour permettre une inter-comparaison de 1 an entre les deux systèmes de mesure.

Pour cette campagne de mesures, des capteurs de pression et de courant ont été déployés en zone intertidale (cf. figure 4). Nous disposions de 8 capteurs de pression piézorésistifs reliés à un datalogger permettant des mesures synchronisées à une fréquence d'échantillonnage de $8 \mathrm{~Hz}$. Ces 8 capteurs de pression étaient déployés suivant deux lignes «cross-shore » parallèles (ligne 1 et 2), comportant chacune 4 capteurs de pression. Nous disposions également d'une troisième ligne composée de 5 capteurs de pression synchronisés à une fréquence d'échantillonnage de $32 \mathrm{~Hz}$. Ces 5 capteurs étaient déployés suivant une ligne «cross-shore », parallèle aux deux précédentes (ligne 3). L'ensemble de ces 13 capteurs de pression était aussi aligné dans la direction parallèle à la plage. Les trois lignes formaient ainsi un rectangle d'environ $150 \mathrm{~m}$ de longueur (longshore) et $50 \mathrm{~m}$ de largeur (cross-shore) et couvraient ainsi un système barre-baïne. Nous disposions également de deux courantomètre-houlographes de type «directionnal Wave Current meter» permettant des mesures de courant (composantes horizontales) et pression à une fréquence de $2 \mathrm{~Hz}$. L'un de ces courantomètres était placé sur la pente de la barre, au niveau de la laisse de basse mer et dans le prolongement de la première ligne de capteurs et l'autre était situé à la naissance de la baïne. Un "Accoustic Doppler Vector» a également été déployé en bas de plage, dans l'alignement de la seconde ligne de capteurs. Il a permis de réaliser des mesures de pression et courant (composantes horizontales et verticales) à des fréquences plus élevées (jusqu'à $32 \mathrm{~Hz}$ ). Un courantomètre Doppler 3500 a également été déployé sur la ligne 3 de capteurs de pression pour mesurer le courant de retour.

La figure 6 illustre la déformation du spectre de densité d'énergie des vagues lorsque l'on se rapproche de la côte. Sur la figure $6 \mathrm{a}$, on note 2 pics correspondant à deux houles distinctes de direction $280^{\circ}$ et $290^{\circ}$ respectivement pour la plus et la moins énergétique. Ces deux modes donnent lieu à des groupement d'ondes qui induisent au large des variations spatio-temporelles de l'énergie (modulation), et éventuellement, après le déferlement, des ondes basses 
fréquences (une analyse des données permettra d'établir si cette hypothèse explique l'énergie des fréquences $<0.05 \mathrm{HZ}$ de la figure $6 \mathrm{~b}$ ).

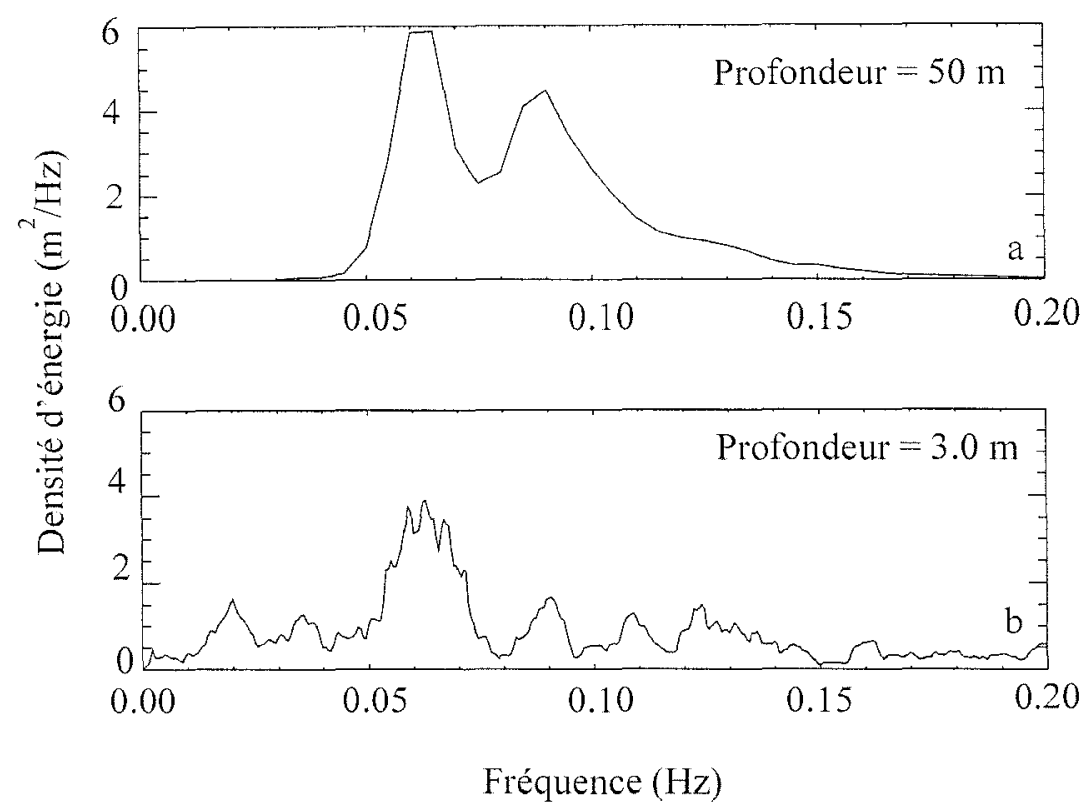

Figure $6:$ Evolution du spectre du large à la zone de surf

Figure 6: Wave spectrum evolution from offshore to the surf zone

Par ailleurs, on distingue nettement une diminution de l'énergie liée au déferlement mais également un élargissement du spectre associé aux transferts d'énergie engendrés par les interactions non-linéaires entre triplets de fréquences.

\section{Mesures hydro-sédimentaires}

Dans la perspective d'accéder au flux sédimentaire en suspension, le LSG a effectué des mesures conjointes d'hydrodynamique et de concentration de sédiment en suspension à l'aide de la station benthique instrumentée SAMBA. Cet équipement composite comprend :

- un module d'acquisition à la cadence de $4 \mathrm{~Hz}$, durant une demi-heure toutes les heures, des données issues d'un capteur de pression (PAROSCIENTIFIC), d'un courantomètre électromagnétique bi-composantes horizontales de $3,8 \mathrm{~cm}$ de diamètre $(\mathrm{MMI})$ et de deux capteurs de matière en suspension à rétrodiffusion infrarouge (OBS-3) ;

- un courantomètre acoustique Doppler tri-composantes incluant un capteur de pression (VECTOR) couplé à un capteur de matière en suspension à rétrodiffusion infrarouge (OBS-3) fonctionnant en continu à la cadence de $8 \mathrm{~Hz}$;

- un multi-échantillonneur séquentiel (toutes les heures) d'eau et de matière en suspension à $0,5 \mathrm{~m}$ au dessus du fond.

La station SAMBA a été déployée en bas de plage (figure 4). A titre d'illustration, la figure 7 montre l'évolution de la concentration de sédiment en 
suspension mesurée par le multi-échantillonneur durant le montant et le perdant de l'après-midi et de la soirée du 16/10/01.

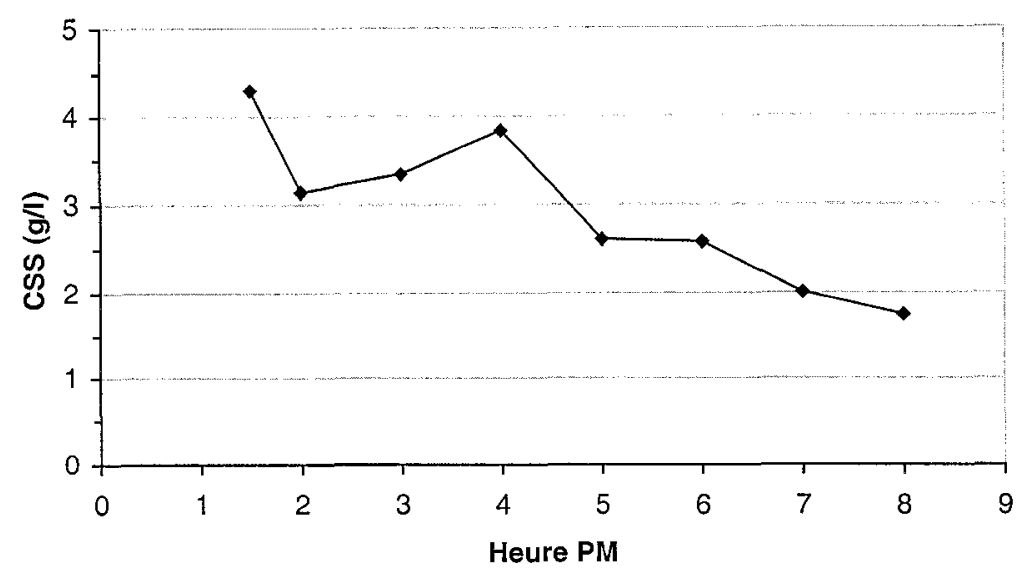

Figure 7. Evolution temporelle de la concentration de sédiment en suspension à $0,5 \mathrm{~m}$ du fond mesurée par le multi-échantillonneur le 16/10/01.

Figure 7. Time-variation of suspended sediment concentration at $0.5 \mathrm{~m}$ above the bottom measured by the multi-sampler on 10/16/01.

\section{Traçage fluorescent}

L'évaluation quantitative du transit sédimentaire sur les estrans sableux a été mise en évidence par la technique du traçage fluorescent. L'utilisation de traceur pour déterminer les taux de transport sédimentaire exige deux conditions :

- le traceur possède le même comportement hydrodynamique que le sédiment naturel, c'est-à-dire les mêmes caractéristiques (forme et taille des grains, densité) - il doit être facilement détectable in situ afin de suivre son déplacement dans l'espace et dans le temps au cours du processus étudié.

Méthodologie : La technique des traceurs fluorescents que nous utilisons correspond à une mesure lagrangienne du transport sédimentaire en zone intertidale. Un Détecteur Compteur Automatique (D.C.A.) de grains fluorescents nous a permis de déterminer le nombre de grains décomptés en surface, en chaque point de la grille d'échantillonnage. L'épaisseur du traceur en mouvement a été, quant à elle, déterminée par un carottage systématique de la zone détectée.

Transport résiduel sur la plage du Truc Vert : $90 \mathrm{~kg}$ de sable fluorescent ont été injectés lors de la basse mer du 17/10 au soir à mi-estran. L'injection s'est faite dans une fosse de $1 \times 1 \times 0.16 \mathrm{~m}$, le traceur étant recouvert de $0.02 \mathrm{~m}$ de sable naturel. La détection s'est effectuée au cours de la basse mer suivante (18/10). Les résultats préliminaires montrent qu'à la source, $0,1 \mathrm{~m}$ d'épaisseur de traceur a été mobilisée lors du flot. Lors du jusant, la source est recouverte par 0,07 $\mathrm{m}$ de sable "propre". Une partie du traceur mobilisé est retrouvée sur la plage sous forme de taches très diluées indiquant une dominance du transport vers le sud (longshore transport) et vers le bas de plage (cross-shore transport). 


\section{VII ${ }^{\grave{m} e s}$ Journées Nationales Génie Civil-Génie Côtier, Anglet, France, 15-17 Mai 2002}

Cette expérience réalisée pendant des conditions hydrodynamiques de forte énergie va nous permettre de définir la direction et la vitesse de transit sableux résiduel au cours d'une marée, mais la forte dilution du traceur ne nous permet pas d'attribuer un degré de confiance suffisant au calcul du débit.

\section{Conclusion}

La campagne de mesures, réalisée sur la plage aquitaine du Truc Vert du 14 au 19 octobre 2001, a permis d'acquérir un jeu de données hydrodynamiques, sédimentaires et topographiques de grande qualité, pour des conditions de forçage de la houle au large très énergétiques. Le couplage de plusieurs techniques expérimentales complémentaires devrait nous permettre d'avancer de façon significative dans la compréhension de la dynamique sédimentaire des plages sableuses soumises à l'action de fortes houles.

Remerciements. Ces travaux ont été soutenus principalement par le Programme National d'Environnement Côtier (PNEC ART7), mais aussi par le Programme ATmosphère Océan à Multi-échelles (PATOM) et l'EPSHOM. Le Cirmat a fourni une partie de l'instrumentation. L'INSU et La Région Aquitaine ont co-financés une partie du matériel. Nous remercions le CETMEF et les phares et Balises du Verdon grâce auxquels la bouée houle TRIAXYS a pu être mise en service. Nous remercions Amaury De Resseguier pour la préparation des structures des courantomètres du DGO et Michel Trilha le pilote de l'avion qui a survolé le site du Truc Vert.

\section{Références}

[1] Bonneton, P. and Dupuis, H. 2001. Transformation of irregular waves in the inner surf zone. Proc. 27th Int. Conf. on Coastal Eng, 1, 745-754.

[2] Butel, R., Dupuis, H. and Bonneton, P. 2001 Spatial and temporal variability of wave conditions at French Atlantic coast using in-situ data. Proc. of ICS2002 submitted to $J$. Coastal Research.

[3] Desmazes, F., Michel, D. et Howa, H. 2001. Etude morphologique et hydrosédimentaire du domaine pré-littoral aquitain. 8ème Congrès Français de Sédimentologie, publication A.S.F. n ${ }^{\circ}$, Paris, 95-96.

[4] Lafon V., Dupuis H., Howa H. et Froidefond J.M.. Determining ridge and runnel longshore morphodynamics using SPOT imagery, Marine Geology (soumis).

15] Michel D. et Howa H. (1999) Short term morphodynamic response of a ridge and runnel system on a mesotidal sandy beach. J. Coastal Research 15/2, 428-437.

[6] Michel D., Howa H., Thomas C., Chapalain G., Thais L. et Malengros D., 2000. Sediment dynamics and hydrodynamics on the inner part of the Aquitanian shelf (France), Workshop Marine Sandwave Dynamics, Lille, 23-25 March 2000.

[7] Sénéchal N., Dupuis H., Bonneton P., Howa H. et Pedreros R. 2001a Observation of irregular wave transformation in the surf zone over a gently sloping sandy beach. Oceanologica Acta (sous presse)

[8] Sénéchal, N., Bonneton, P. et Dupuis, H., 2001b. Field experiment on secondary wave generation on a barred beach and the consequent evolution of energy dissipation on the beach face. soumis à Coastal Engineering. 